\title{
LAS PANTALLAS ACÚSTICAS COMO SOLUCIÓN A LA CONTAMINACIÓN SONORA EN EL PARADERO BENAVIDES
}

\author{
ACOUSTIC SCREENS AS A SOLUTION \\ TO NOISE POLLUTION IN THE BENAVIDES STOP
}

Yeampier Witming Iñigo Pozo ${ }^{1}$, Lucero Romero Santos ${ }^{2}$,
Esther Vargas Chang ${ }^{3}$

RECEPCIÓN: 24 DE AGOSTO DE 2021

ACEPTACIÓN: 10 DE SETIEMBRE DE 2021

\section{RESUMEN}

En Perú no es común el uso de barreras acústicas como tecnología para disminuir el exceso de contaminación sonora en distintos lugares del país. Por este motivo el objetivo de esta investigación es proponer el uso de pantallas acústicas como una medida para reducir la contaminación sonora en el paradero Benavides. Las pantallas pueden ser de hormigón, metálicas, madera y vegetal siendo los más comerciales y con mayor índice de reducción de decibeles. La investigación es de tipo descriptiva basada en la recolección de datos e interpretación de los mismos a fin de comparar las diferentes tipologías de pantallas acústicas y analizar su factibilidad de uso en comparación con proyectos similares en los cuales haya sido empleada esta novedosa tecnología. Es importante destacar que las barreras acústicas son una tecnología muy empleada en Europa. No obstante, a nivel nacional y en todo Sudamérica aún no han sido puestas en práctica. Incluso Perú no cuenta con una normativa que indique su uso y diseño para emplearla en proyectos. Esto limita a hacer uso de esta solución. Sin embargo, podría implementarse debido a los buenos resultados obtenidos.

Palabras clave: Pantalla acústica, contaminación sonora, barreras antirruido.

\begin{abstract}
In Peru it is not common to use noise barriers as a technology to reduce excess noise pollution in different parts of the country. For this reason, the objective of this research is to propose the use of acoustic screens as a measure to reduce noise pollution in the Benavides whereabouts.

The screens can be made of concrete, metal, wood and vegetable being the most commercial and with the highest rate of reduction of decibels. The research is descriptive based on data collection and interpretation of the same in order to compare the different typologies of acoustic screens and analyze their feasibility of use compared to similar projects in which this novel technology has been used.

It is important to note that noise barriers are a widely used technology in Europe. However, at the national level and throughout South America it has not yet been implemented. Even Peru does not have a regulation that indicates its use and design to use it in projects. This limits to make use of this solution. However, it could be implemented due to the good results obtained.
\end{abstract}

Keywords: Acoustic screens, noise pollution, antinoise barriers

\footnotetext{
1 Universidad Ricardo Palma. Facultad de Ingeniería. <yeampier.inigo@urp.edu.pe>

2 Universidad Ricardo Palma. Facultad de Ingeniería. <lucero.romeros@urp.edu.pe>

3 Universidad Ricardo Palma. Facultad de Ingeniería. <esther.vargas@urp.edu.pe>
} 


\section{INTRODUCCIÓN}

La contaminación sonora demostró ser un problema en la actualidad en la ciudad Metropolitana de Lima debido a la circulación excesiva de vehículos. En ese sentido, en el Paradero Benavides se concentra un alto índice de ruido, por lo que se propone el uso de pantallas acústicas para así disminuir el nivel de contaminación.

En el artículo "Contaminación sonora en el paradero Benavides, en la ciudad de Lima y la afectación en poblaciones aledańas (horario diurno)" realizada por alumnos de la Universidad Ricardo Palma, se realizaron mediciones diarias en las cuales se capturaron los sonidos que se producen y que son causados por fuentes vehiculares de transporte público y privado. Estos sonidos constituyen altos niveles de contaminación sonora. Sobre la base de la información previamente citada, se confirma la necesidad de una solución inmediata que ayude a disminuir el número de decibeles en el paradero Benavides, puesto que es un punto de alta concentración de ruido.

Por eso, se busca proponer una solución práctica, viable y económicamente accesible que garantice un adecuado número de decibeles emitidos. Se eligió el uso de pantallas acústicas ante esta problemática, porque estas ayudan a controlar la propagación del ruido y la reverberación, lo que disminuye el nivel sonoro y crea un entorno saludable y sostenible. El resultado de esta investigación será de utilidad para mejorar la calidad de vida de las personas expuestas al ruido excesivo producido en el paradero Benavides y busca crear un clima adecuado acorde al nivel indicado en el reglamento peruano.

\section{OBJETIVOS}

Objetivo General:

- Proponer el uso de pantallas acústicas como una medida para reducir la contaminación sonora en el paradero Benavides.

Objetivos específicos:

- Describir la existencia de la contaminación sonora en el paradero Benavides.

- Describir la reducción de ruido al instalar pantallas acústicas.

- Describir los resultados de esta tecnología aplicada en construcciones nacionales e internacionales.

- Analizar la viabilidad del uso de los paneles acústicos en el paradero Benavides.

\section{FUNDAMENTO TEÓRICO}

\section{El ruido}

De acuerdo con Puma [1], es un sonido no deseado con carácter subjetivo, considerado mayormente como molestia y es denominado contaminante al ser una perturbación en el ambiente ocasionando daño o lesión en el oído humano.

\section{Generación y transmisión del sonido}

Se necesitan tres elementos para que exista el sonido: una fuente que sea capaz de producir una perturbación mecánica, un medio en el cual se propague la perturbación, y un receptor donde la perturbación produce una sensación auditiva.

\section{Contaminación sonora}

La contaminación sonora es causada por ruidos y vibraciones en el ambiente, lo que produce molestia, riesgo o daño para las personas y genera efectos negativos en el medio ambiente [1, p. 12] 


\section{Ruido de tráfico}

De acuerdo con investigaciones realizadas la fuente principal de contaminación son los vehículos a motor, pues más del $80 \%$ del ruido es causado por el tráfico. Debido a que la vida urbana es un poco compleja, existe la necesidad de aumentar su movilidad lo que incrementa el uso de vehículos y, los ruidos del ruido del motor, estado de conservación aumentan el nivel sonoro. [1, p. 13].

\section{Posibles consecuencias}

La contaminación sonora produce algunas consecuencias como:

a) Fatiga auditiva

b) Pérdida de atención, concentración y rendimiento

c) Falta de sueño

\section{Normativa y legislación ambiental}

\section{Normativa nacional}

El Perú cuenta con normativas que logran una correcta gestión de ruido ambiental:

a) La Constitución Política del Perú ${ }^{1}$, en su artículo 2, inciso 22, señala que "Toda persona tiene derecho a la paz, a la tranquilidad, el disfrute del tiempo libre y al descanso, así como gozar de un ambiente equilibrado y adecuado del desarrollo de su vida."

b) Ley General del ambiente $\mathrm{N}^{\circ} 28611^{2}$ en su artículo 115 señala que es responsabilidad de los gobiernos locales "dictar normas y controlar los ruidos y vibraciones que sean originados por las actividades domésticas y comerciales, así como también por las fuentes móviles, debiendo instaurar la normativa respectiva sobre la base de los Estándares de Calidad Ambiental".

c) El Decreto Supremo No 085-2003-PCM - Reglamento de Estándares Nacionales de Calidad Ambiental para Ruido ${ }^{3}$ establece que "Representan los máximos niveles de ruido en el ambiente que no deben ser sobrepasados para garantizar la protección de la salud humana, según cuatro zonas de aplicación”.

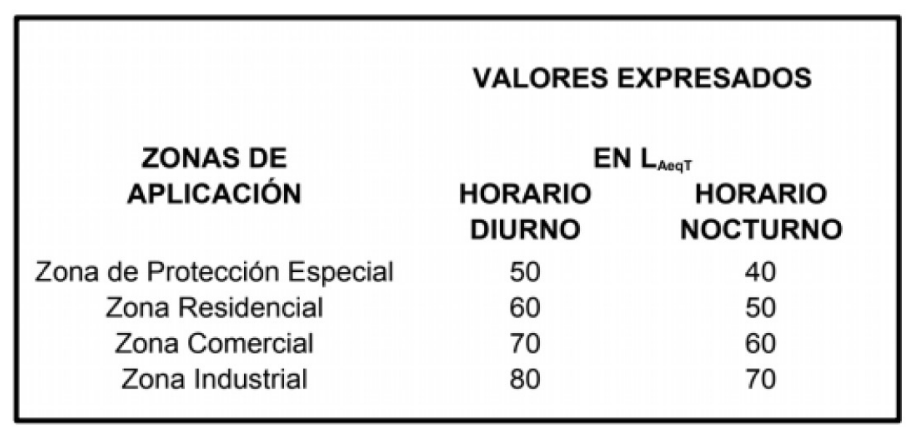

Figura 1. Estándares de Calidad Ambiental para Ruido por cada Zona de Aplicación.

Fuente: El Peruano Normas Legales (2003)

1 Constitución política del Perú, 1993.

2 Ley General del Ambiente No 28611, promulgada el 13 de octubre 2005.

3 Reglamento de Estándares Nacionales de Calidad Ambiental para Ruido. Decreto de 30 de octubre 2003. DS No 0852003-PCM 
d) El Protocolo Nacional de Monitoreo de Ruido Ambiental AMC N031-2011- MINAM/ $\mathrm{OGA}^{4}$ establece unos límites máximos de ruido que se pueden permitir a nivel urbano y señala que son las municipalidades distritales las que deben desarrollar normas y medidas concretas para evitar y controlar la contaminación auditiva severa.

\section{Normativa internacional}

De acuerdo con Asebal [2] la Norma UNE EN 14388 establece un conjunto de obligaciones específicas del fabricante:

1. Proporcionar las instrucciones de instalación, que deben describir el modo en que el producto (elemento acústico, pantalla anti ruido completa, etc.) debe instalarse para poder obtener el comportamiento medido en el ensayo de tipo inicial y un manual de mantenimiento que debe especificar las medidas que son necesarias; o que deben evitarse, para mantener la durabilidad del comportamiento acústico, la transparencia, la resistencia estructural, etc.

2. Realizar ensayos iniciales en la primera aplicación de esta norma.

3. El fabricante debe establecer, documentar y mantener un sistema de Control de Producción en Fábrica (CPF) para asegurar que los productos comercializados son conformes con las características de comportamiento indicadas. Debe considerarse que un sistema de CPF conforme a la Norma EN ISO 9001:2000 y particularizado para los requisitos de esta norma satisface los requisitos.

Por su parte, de acuerdo con Asebal [3], la Norma UNE EN 1793-1, "Características relativas a la absorción acústica”, señala que el método de laboratorio:

permite medir la absorción acústica de las pantallas anti ruido planas o de los revestimientos planos para muros de contención o túneles. Se utiliza para evaluar el comportamiento de absorción acústica intrínseco de los dispositivos reductores de ruido de tráfico en carreteras que puedan montarse de forma razonable dentro de las instalaciones de ensayo descritas en la Norma EN 20354. Los resultados aportan una tabla y un gráfico que reflejan todas las frecuencias de medición de absorción acústica $\alpha_{\mathrm{Si}}$ para cada cambio de frecuencia durante el ensayo.

\begin{tabular}{cc}
\hline \multicolumn{2}{c}{ Categorías de comportamiento de absorción } \\
\hline Categoría & $\mathrm{Db}$ \\
\hline $\mathrm{A} 0$ & No determinado \\
\hline $\mathrm{A} 1$ & $<4$ \\
\hline $\mathrm{A} 2$ & $4 \mathrm{a} 7$ \\
\hline $\mathrm{A} 3$ & 8 a 11 \\
\hline $\mathrm{A} 4$ & $>11$ \\
\hline
\end{tabular}

Tabla 1. Categorías de comportamiento de absorción. Fuente: Norma UNE EN 1793-1

En esa misma línea, Asebal [4] plantea que la Norma UNE EN 1793-2, "Características intrínsecas relativas al aislamiento al ruido aéreo" establece que:

el método de laboratorio que permite medir el comportamiento de aislamiento al ruido aéreo de las pantallas anti ruido en las carreteras. Se utiliza esta norma para la evaluación del comportamiento intrínseco de las pantallas anti ruido que se pueden ensamblar de forma razonable dentro de las insta- 
laciones de ensayo descritas en la norma EN ISO 140-3.

Los resultados aportan una tabla y un gráfico que refleja los índices de aislamiento acústico Ri para todas las frecuencias ensayadas. El índice de evaluación del aislamiento al ruido aéreo; DLR, es el que se obtiene redondeando al entero más cercano. Si además del índice se quiere clasificar el comportamiento del aislamiento; la norma prevé la siguiente tabla:

\begin{tabular}{cc}
\hline \multicolumn{2}{c}{ Categorías de comportamiento de aislamiento } \\
\hline Categoría & Db \\
\hline B0 & No determinado \\
B1 & $<15$ \\
B2 & 15 a 24 \\
B3 & $>24$ \\
\hline
\end{tabular}

Tabla 2. Categorias de comportamiento de aislamiento.

Asimismo, según Asebal [5], la Norma UNE EN 1794-1, "Comportamiento mecánico y requisitos de estabilidad", mide:

los esfuerzos mecánicos a los que se ven sometidas las pantallas en su ubicación. Las deformaciones de un dispositivo reductor de ruido bajo estas cargas durante su vida útil de diseño no deberían reducir su eficacia.

1) Apartados de la norma:

2) Carga de viento y carga estática

3) Vibración y efectos de fatiga

4) Peso propio

5) Impacto de piedras

6) Seguridad en caso de colisión

Cargas dinámicas ocasionadas por la retirada de la nieve

Cuando los valores obtenidos en cada uno de los apartados anteriores son de acuerdo a [sic] la norma, se considera que la pantalla cumple con el comportamiento mecánico y estabilidad suficiente para ser instalada en su emplazamiento.

Finalmente, Asebal [6] plantea que la Norma UNE EN 1794-2, "Requisitos en relación con la seguridad general y el medio ambiente", establece los siguientes:

requisitos para todos los elementos instalados en cualquier carretera; que además de desempeñar su función, no representen ningún riesgo para los usuarios de la carretera ni para las demás personas que pudieran estar cerca, o para el medio ambiente en general.

Estos dispositivos no deberían facilitar la propagación de un incendio desde el arcén o los terrenos colindantes. Puede ser exigible; además, una resistencia al fuego que cumpla con normas particulares para reducir el riesgo de propagación hacia los locales contiguos o hacia los usuarios de la carretera en corredores estrechos.

Los dispositivos reductores de ruido de tráfico por carretera no deberían reflejar la luz hasta el punto de perjudicar la seguridad vial. Los materiales con los que están fabricados los dispositivos reductores de ruido no deberían; bajo ningún concepto, emitir humos o líquidos nocivos procedentes de procesos naturales o industriales, o bien provocados por un incendio. Estos dispositivos deberían permitir que los usuarios de la carretera puedan salir rápidamente y facilitar así mismo el acceso del personal de seguridad en caso de emergencia.

Esta norma europea especifica los requisitos mínimos y otros criterios de evaluación de la seguridad 
CIVIL

general, así como el comportamiento en relación con el medio ambiente de los dispositivos reductores de ruido de tráfico por carretera, bajo las condiciones típicas existentes junto a las carreteras.

\section{Barreras acústicas}

Quirós [7] define las pantallas o barreras acústicas como una estructura sólida capaz de reducir los niveles entre la fuente y receptor, actuando con respecto a la onda incidente difractándola, proporcionando diferentes atenuaciones a diferentes frecuencias creando una sombra acústica.

De acuerdo con el modelo, el grado de absorción de acústica-carretera y acústica-tren puede medirse de la más débil (A1) a la más fuerte (A5). Por otro lado, su grado de aislamiento puede medirse de la más débil (B1) a la más fuerte (B5).

\section{Tipos de barreras acústicas}

a) Pantallas acústicas metálicas

Las pantallas acústicas metálicas están formadas por dos láminas de metal. Estas suelen ser normalmente de acero y cuentan con un núcleo de lana mineral entre ambas.

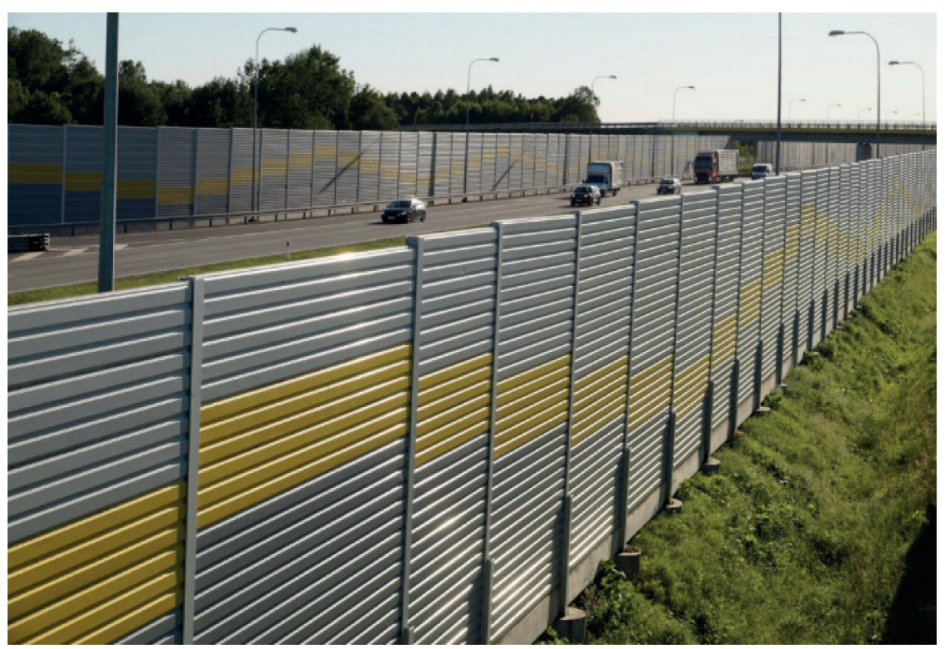

De acuerdo con el portal de Insametal [8], este tipo de pantalla "proporcionan la durabilidad y resistencia que la mayoría de nuestros clientes desean. El material es más ligero que el hormigón y la resistencia es parecida." 


\begin{tabular}{lll}
\hline Modelo & Acústica-Carretera & Acústica-Tren \\
\hline P57 & A4/B2 & - \\
P58 & A4/B3 & $14 \mathrm{~dB} / 30 \mathrm{~dB}$ \\
P68 BOBLE ABSOR. & & \\
P68 DOBLE ABSOR. + AISLAMIENTO & A3/B2 & - \\
P68 PLUS & & \\
P68 MEGA & & \\
P81 & A4/B3 & \\
P81 MEGA & & - \\
INSAVE 4.0 & & \\
& A4/B3 & $14 \mathrm{Db} / 30 \mathrm{~dB}$ \\
& A4/B3 & $14 \mathrm{~dB} / 30 \mathrm{~dB}$ \\
& A4/B3 & - \\
& A4/B3 & - \\
& A3/B3 & $8 \mathrm{~dB} / 26 \mathrm{~dB}$ \\
\hline
\end{tabular}

Tabla 3. Clasificación acústica de Pantalla metálica. Fuente: INSAMETAL

b) Pantallas acústicas de hormigón

El mencionado portal de Insametal, señala, a propósito de este tipo de apantallamiento, que:

Se componen de una estructura de hormigón armado, la cual cuenta con varios centímetros de espesor revestida por dos capas de conglomerado de madera mineralizada y cemento. En sus extremos se cuenta con una banda maciza de hormigón, la cual le permite tener la facilidad de encajar en cualquier tipo de viga de acero de perfiles estándar.

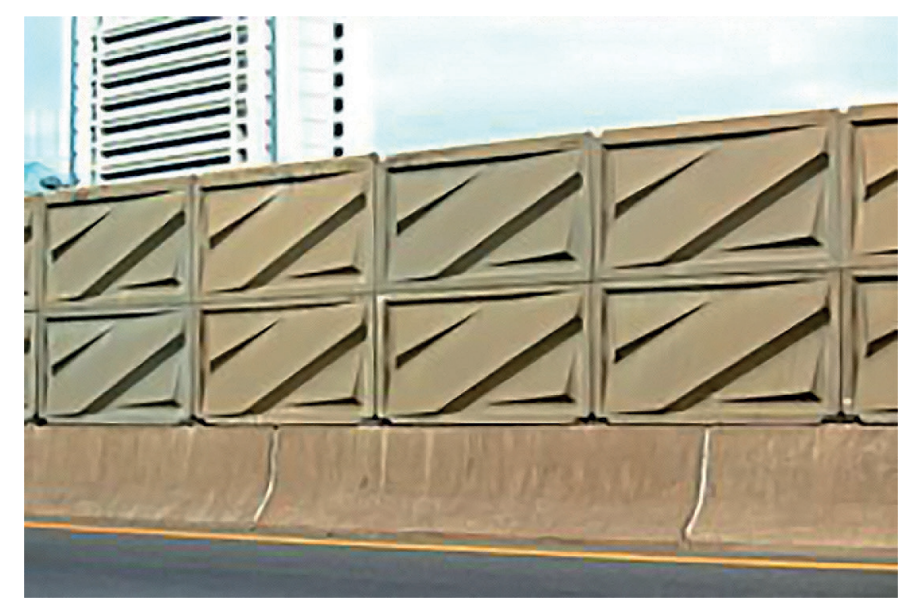

Figura 3. Pantalla acústica de hormigón. Fuente: Paneles ACH.

\begin{tabular}{cc}
\hline Modelo & Acústica-Barrera \\
\hline INSA Rock 1 & A2/B3 \\
INSA Rock 2 &
\end{tabular}

A3/B3

Tabla 4. Clasificación acústica de Pantalla de hormigón. Fuente: INSAMETAL 
CIVIL

c) Pantallas de madera

Este tipo de barreras acústicas son construidas íntegramente en madera y su uso es habitual en zonas anexas a vías pecuarias.

Se cuenta con los posibles formatos:

- Tablas de madera de pino CL4 tratada a la autoclave.

- Rollizos de madera de pino CL4 tratada a la autoclave.

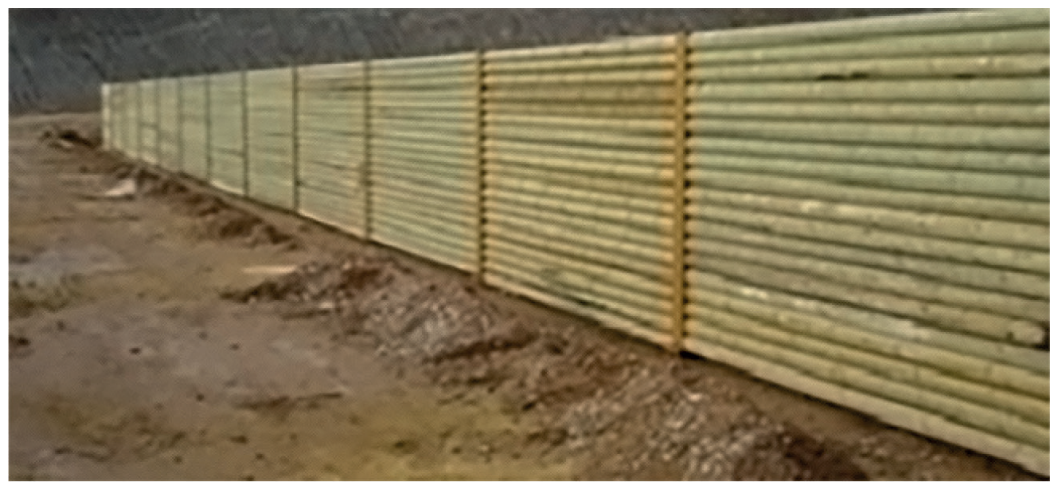

Figura 4. Pantalla de madera. Fuente: Paneles ACH

\begin{tabular}{cc}
\hline Modelo & Acústica-Barrera \\
\hline Kasttor & A2/B3
\end{tabular}

Tabla 5. Clasificación acústica de Pantalla de madera. Fuente: INSAMETAL

\begin{tabular}{ll}
\hline \multicolumn{2}{c}{ COMPORTAMIENTO GENERAL } \\
\hline CARACTERÍSTICAS MECÁNICAS & VALOR \\
\hline Longitud = $4 \mathrm{~m}$ & $177 \mathrm{~kg} / \mathrm{m} 2$ \\
Carga máxima horizontal hasta $\mathrm{f}>50 \mathrm{~mm}$ & $239 \mathrm{~kg} / \mathrm{m} 2$ \\
Carga máxima horizontal sin rotura & $341 \mathrm{~kg} / \mathrm{m} 2$ \\
Carga de rotura & VALOR \\
\hline PROPIEDADES DE LA MADERA & Certificado PEFC \\
\hline Sistema de control de la cadena de custodia de productos forestales & Nivel “clase IV" en autoclave, al \\
Tratamiento para exteriores & vacío y por inyección \\
\hline CARACTERÍSTICAS ACÚSTICAS & VALOR \\
\hline Índice de absorción DL & 8 db (A) \\
Índice de aislamiento global DL & $29 \mathrm{db}(\mathrm{A})$ \\
\hline CARACTERÍSTICAS DIMENSIONALES & VALOR \\
\hline Longitud & Hasta $4000 \mathrm{~mm}$ \\
Ancho & Hasta $2000 \mathrm{~mm}$ \\
Espesor & $95 \mathrm{~mm}$ \\
\hline
\end{tabular}

Tabla 6. Caracteristicas de Pantalla acústica de madera PANACOR MD11. Fuente: INSAMETAL 
d) Pantallas Vegetales

Son aquellas barreras realizadas a partir de estructuras metálicas que alojan en su entramado elementos que posibilitan el desarrollo de especies vegetales como reciclados, desperdicios de alfombras, restos de papel, materiales fibrosos, deshechos de jardines, grava etc.

Según Serrano et al. [9], su mecanismo de funcionamiento se basa en que los niveles de ruido producido por el tráfico pueden reducirse por absorción y difusión del sonido por la vegetación. Para conseguir una aceptable reducción del ruido la vegetación debe ser alta motivo por el cual se considera una escasa efectividad.

Figura 5. Pantallas vegetales. Fuente: Paneles ACH.

\begin{tabular}{cc}
\hline Modelo & Acústica-Barrera \\
\hline Vegetal & A4 \\
\hline
\end{tabular}

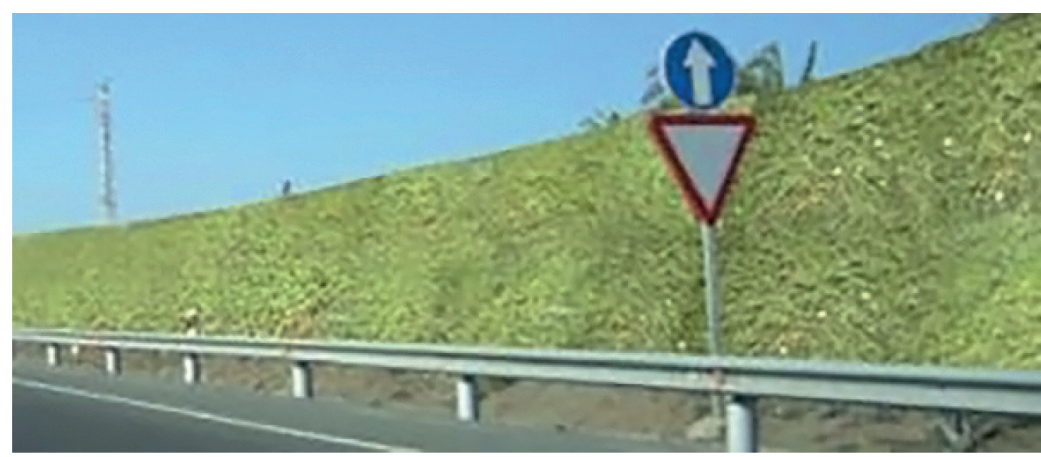

Tabla 6. Clasificación acústica de Pantalla vegetal. Fuente: Paneles ACH

\begin{tabular}{cccc}
\hline ACH & $\begin{array}{l}\text { Aislamiento } \\
\text { acústico }\end{array}$ & Absorción acústica & Peso/m2 del panel \\
\hline Metálica & B3 & A3-A4 & $20 \mathrm{~kg}$ \\
\hline Hormigón & B3 & A2-A3 & Hasta 350 kg \\
& & \\
\hline
\end{tabular}

Tabla 7. Características de Pantalla Metálica y Pantalla de hormigón. Fuente: INSAMETAL

Se debe entender que el grado de absorción puede medirse de la más débil (A1) a la más fuerte (A5). Por otro lado, su grado de aislamiento puede medirse de la más débil (B1) a la más fuerte (B5).

En la Tabla 6, se puede observar que la pantalla vegetal presenta un grado de absorción A4. Sin embargo, no cuenta con un grado de aislamiento lo que genera cierta desventaja en comparación con las pantallas metálicas y de hormigón.

$\mathrm{Al}$ evaluar la mejor propuesta entre pantallas metálicas y de hormigón, se recomienda utilizar las primeras pues en la Tabla 7 se observa un mayor de grado de absorción. Asimismo, al considerar el 
peso/m2 se observa una gran diferencia pues en las pantallas de hormigón se aproxima hasta $350 \mathrm{~kg} /$ m2 mientras que las metálicas $20 \mathrm{~kg}$. Esta situación implicaría una gran diferencia de costos, lo cual determinaría como mejor opción a las pantallas metálicas.

\section{CONCLUSIONES}

Con el trabajo realizado se concluye que las pantallas acústicas son una alternativa de solución contra el problema de la contaminación sonora en el paradero Benavides, ya que son utilizadas en otros países con fines similares a los que se dirige la investigación. Queda claro que traen consigo un alto nivel de eficacia y confiabilidad, y brindan mejores estándares de calidad ambiental y cuidado de la salud en las personas.

Resulta evidente la presencia de contaminación sonora en el paradero Benavides debido a los altos índices de decibeles expuestos en artículo "Contaminación sonora en el paradero Benavides en la ciudad de Lima y la afectación en poblaciones aledańas horario diurno" [10]. De esta forma, se demuestra la necesidad de disminuir los elevados índices que causan daños en el ambiente y en la salud de las personas que transitan a diario por esta zona.

Se logró identificar los diferentes tipos de pantallas acústicas que existen, se señalaron diversas características de estas, como sus características mecánicas, propiedades del material, acústicas y dimensionales.

Las investigaciones encontradas tanto nacionales como internacionales, al ser estudios cuantitativos, demuestran una reducción de decibeles de acuerdo con el material con el que se realizaron las pruebas.

Se puede concluir la viabilidad existente al emplear esta tecnología debido a que existen características acústicas, un índice de absorción y de aislamiento. Los resultados demuestran una disminución de los decibeles en un ambiente de tránsito y circulación lo que ha permitido alcanzar resultados positivos.

\section{RECOMENDACIONES}

Las pantallas acústicas son una tecnología novedosa que es empleada en el continente europeo a fin de disminuir los niveles de contaminación acústica y buscan generar un mejor ambiente. Por ello, implementarlas en Perú y en todo Sudamérica sería una excelente alternativa para disminuir la contaminación tanto ambiental como acústica. Es de gran importancia implementar una normativa nacional que contemple el uso y diseńo de pantallas acústicas para ser utilizadas en futuros proyectos. Debido a la ausencia de una normativa adecuada, el uso de esta tecnología se encuentra limitada en Perú. Países europeos cuentan con normativas que estipulan criterios para emplear pantallas acústicas $y$, por ello, su uso es masivo y se encuentran presentes en proyectos de gran envergadura a fin de cuidar sus estándares ambientales y proteger la salud de las personas.

\section{REFERENCIAS BIBLIOGRÁFICAS}

[1] J. Puma, "Atenuación sonora por barreras acústicas a base de residuos orgánicos para reducir el nivel de ruido en una avenida principal, Puente Piedra, 2018", tesis de pregrado, Universidad César Vallejo, 2018 [En línea]. Disponible en: https://bit.ly/3pF0sqB [Accedido: 30-dic-2021] 
[2] Resumen UNE EN 14388 "Dispositivos de reducción de ruido de tráfico de carreteras. Especificaciones", Asebal [En línea]. Disponible en: https://bit.ly/3FGHaXf [Accedido: 30-dic-2021]

[3] Resumen UNE EN 1793-1 "Características relativas a la absorción acústica", Asebal [En línea]. Disponible en: https://bit.ly/32QnVfz [Accedido: 30-dic-2021]

[4] Resumen UNE EN 1793-2 "Características intrínsecas relativas al aislamiento al ruido aéreo", Asebal [En línea]. Disponible en: https://bit.ly/32Floox [Accedido: 30-dic-2021]

[5] Resumen UNE EN 1794-1 “Comportamiento mecánico y requisitos de estabilidad”, Asebal [En línea]. Disponible en: https://bit.ly/3JmXK0C [Accedido: 30-dic-2021]

[6] Resumen UNE EN 1794-2 "Requisitos en relación con la seguridad general y el medio ambiente", Asebal [En línea]. Disponible en: https://bit.ly/3qxSEpT [Accedido: 30-dic-2021]

[7] R. Quirós, "Estudio de pantallas acústicas elaboradas a partir de green composites", tesis de maestría, Universidad Politécnica de Valencia, 2013 [En línea]. Disponible en: https://bit.ly/3sLqQkG [Accedido: 30-dic-2021]

[8] "Pantalla Acústica Metálica", Insametal, 2021. [En línea]. Disponible en: https://insametal.es/ pantalla-acustica-metalica/ [Accedido: 30-dic-2021]

[9] M. Serrano, L. Abad, R. Magro, y T. García, "Estudio de la tipología de las pantallas acústicas. Normativa y estado del arte”, Tecnología y desarrollo, vol. 7, pp. 1-44, 2009 [En línea]. Disponible en: https://bit.ly/3ezCh6v [Accedido: 30-dic-2021]

[10] L. Romero, D. Bravo, y C. Tufiño, "Contaminación sonora en el paradero Benavides en la ciudad de lima y la afectación en poblaciones aledañas (horario diurno)”, 2017 [En línea]. Disponible en: https://bit.ly/33YQgAG [Accedido: 30-dic-2021] 